\title{
SPIN-ORBIT COUPLING FOR $f$-ELECTRONS IN A CRYSTALLINE FIELD
}

\author{
T. LULEK \\ Institute of Physics, Adam Mickiewicz University \\ Matejki 48/49, 60-769 Poznań, Poland
}

The notion of a spin-orbit interaction arises from consideration of $\mathbf{d y}$ namics of multielectron atoms, i.e. systems of $N$ electrons in a spherical potential. This notion is essentially a single-particle one. We sketch its origin as a second-order correction when Dirac four-component wave equations for an electron in external electromagnetic fields are simplified to the two-component Pauli spinors. The constraints in spinorial degrees of freedom consist, roughly speaking, in neglecting the small component of the electron four-function. The spin-orbit interaction term serves to compensate effects of the small component. The crystalline field induces some deviations from spherical symmetry of an isolated atom, which yields some modifications of the spherical form of the spin-orbit interaction operator. These modifications can be described in terms of a number of tensor operators adapted to appropriate chains of subgroups of the spherical symmetry group. We present a classification of independent tensor operators and discuss the relevant parameters for $f$-ions.

PACS numbers: 71.70. Ch

\section{Introduction}

The aim of the present article is to provide a review of some notions related to the spin-orbit interaction in $f$-electron systems. First we explain the origin of this interaction as a result of simplification of the exact Dirac four-component wave equation for an electron in an external electric field to the two-component Pauli theory, used extensively in the quantum theory of multielectron atoms [1]. Then we describe a classification of independent radial parameters for a complete presentation of effects of the spin-orbit interaction in a crystalline field, which breaks the spherical symmetry of the central field of an atom to the point symmetry of its crystal environment $[2,3]$. 


\section{Origin of the spin-orbit interaction}

Experimental foundations of a spin-orbit coupling arose in the very early stage of interpretation of the fine structure of atomic spectra. A typical energy level of a multielectron atom is characterized by $L S$ quantum numbers which describe the orbital and spin angular momentum of the electronic shell. For a sufficient spectroscopic resolution, this level splits into $2 S+1$ sublevels, characterized by the total angular momentum $J$ of the shell. The structure of this splitting is attributed to the spin-orbit interaction, which is given - within $L S$ Russel-Saunders term - by a simple Hamiltonian

$$
\widehat{\mathcal{H}}_{\text {so }}=\lambda \widehat{\boldsymbol{L}} \cdot \widehat{\boldsymbol{S}}
$$

where $\widehat{\boldsymbol{L}}$ and $\widehat{\boldsymbol{S}}$ are, respectively, orbital and spin angular momentum operators, and $\lambda$ describes the strength of the interaction. Using an elementary formula

$$
(\widehat{\boldsymbol{L}}+\widehat{S})^{2}=J(J+1)
$$

where the last equality holds only within the manifold of all states of the $L S$ term, one gets

$$
\hat{\mathcal{H}}_{\text {so }}=\frac{\lambda}{2}[J(J+1)-L(L+1)-S(S+1)],
$$

which, in particular, yields the Lande rule

$$
\Delta E=E_{J}-E_{J-1}=\lambda J
$$

for energy separation between consecutive fine energy levels $E_{J}$.

Such a solid experimental basis seems to suggest that the spin-orbit coupling belongs to the principal interactions in the nature. Actually, in the light of present stage of quantum theory, it looks that the spin-orbit coupling is an effect of some approximations rather than a real interaction. Moreover, it should not be attributed to as a purely "relativistic" or "quantum" effect, but emerges also from theories based on Galilean group $[4,5]$ and classical theory (like Thomas precession $[6,7])$. In this paper, however, we shall follow a traditional Lorentzian relativity and the quantum Dirac equation. Let

$$
\widehat{\mathcal{H}}_{\mathrm{D}}=c \boldsymbol{\alpha} \cdot \boldsymbol{p}+\beta m c^{2}+V
$$

be the Dirac Hamiltonian for an electron in an external electrical field of the potential $V$, with

$$
\alpha=\left(\begin{array}{cc}
0 & \sigma \\
\sigma & 0
\end{array}\right), \quad \beta=\left(\begin{array}{cc}
I & 0 \\
0 & -I
\end{array}\right),
$$

being the standard Dirac matrices, where $\sigma$ is the Pauli $2 \times 2$ vector matrix, $I-$ the unit $2 \times 2$ matrix, $m$ - the rest mass of the electron, and $c-$ the velocity of light. The eigenproblem of the Hamiltonian $\widehat{\mathcal{H}}_{\mathrm{D}}$ reads

$$
\widehat{\mathcal{H}}_{\mathrm{D}} \Psi=E \Psi
$$

where

$$
\Psi=\left(\begin{array}{l}
\phi \\
\chi
\end{array}\right)
$$


is a four-component Dirac eigenstate of energy $E$, with $\phi$ and $\chi$ being the large and small components, respectively. Introducing the "Schrödinger energy" $W$ by

$$
E=m c^{2}+W
$$

one rewrites the eigenproblem (7) in the form

$$
\begin{aligned}
& (V-W) \phi+c \boldsymbol{\sigma} \cdot \boldsymbol{p} \chi=0, \\
& \boldsymbol{c} \boldsymbol{\sigma} \cdot \boldsymbol{p} \phi+\left(V-W-2 m c^{2}\right) \chi=0 .
\end{aligned}
$$

From Eq. (10b), the small component is

where

$$
\chi=\frac{1}{2 m c^{2}} f_{w}(r) \boldsymbol{\sigma} \cdot \boldsymbol{p} \phi
$$

$$
f_{w}(r)=\left(1-\frac{V-W}{2 m c^{2}}\right)^{-1}=\sum_{k=0}^{\infty}\left(\frac{V-W}{2 m c^{2}}\right)^{k}
$$

is a real function of position $r$, with the values in a proximity of 1 for sufficiently low energies $W\left(|V-W| \ll 2 m c^{2}\right)$.

A transition from the Dirac to Pauli theory of an electron consists in replacing the Dirac Hamiltonian $\mathcal{H}_{\mathrm{D}}$ and the four-component wave function $\Psi$ by a Pauli Hamiltonian

$$
\mathcal{H}_{\mathrm{P}}=\frac{p^{2}}{2 m}+V+\sum_{k=1}^{\infty} \mathcal{H}_{\mathrm{P}}^{(k)}
$$

and the large component $\phi$ as the corresponding two-component wave function. The term $k=0$ in the expansion (12) corresponds to the Schrödinger Hamiltonian $\widehat{p}^{2} / 2 m+V$, whereas the term with $k=1$ yields the following corrections

$$
\mathcal{H}_{\mathrm{P}}^{(1)}=\frac{1}{4 m^{2} c^{2}} p^{2}(V-W)+\frac{\hbar^{2}}{8 m^{2} c^{2}} \Delta V+\frac{\hbar^{2}}{4 m^{2} c^{2}} \sigma \cdot(\operatorname{grad} V \times p) .
$$

The first form in Eq. (14) corresponds to the effect of dependence of mass upon electron velocity, the second is the Darvin trembling term related to virtual creation and anihilation processes in the vicinity of a singularity of the electric field, and the third describes the spin-orbit coupling.

Chatterjee and Lulek [1] examined a question whether the third term in Eq. (14) is the most general form of spin-dependent energy of an electron within the Pauli theory. To this aim, they considered the Pauli eigenproblem in a form

$$
\tilde{\mathcal{H}}_{\mathrm{P}} \psi_{\mathrm{P}}
$$

where

$$
\psi_{\mathrm{P}}=R \Psi
$$

is the normalized two-component Pauli function, $R$ is a singular operator which has to reduce some discrete degrees of freedom of the Dirac description, and

$$
\tilde{\mathcal{H}}_{\mathbf{P}}=R \mathcal{H}_{\mathrm{P}} R^{-1} \text {. }
$$

The simplest choice is

$$
R_{0}=\left(\begin{array}{ll}
I & 0 \\
0 & 0
\end{array}\right),
$$


which selects the large component $\varphi$ as the Pauli eigenstate $\psi_{\mathrm{P}}$, but it is cleary not a unique possibility, and, moreover, it does not preserve the normalization with the required accuracy (for $k=1$ in the expansion (12)). One can allow for an admixture of a small component $\chi$, but it yields some difficulties with proper account of parity.

We recall that the parity operator $I$ for the electron in Dirac theory is factorised as

$$
I=\beta I_{0},
$$

where $\beta$, given by Eq. (6), is the intrinsic parity of Dirac field, imposed by the corresponding irreducible representation $D^{(1 / 2,0)} \bigoplus D^{(0,1 / 2)}$ of the full Lorentz group, and $I_{0}$ is the orbital parity. Their actions on Dirac wave functions are given by

$$
\begin{aligned}
& \beta \Psi_{\mathrm{D}}=\beta\left(\begin{array}{l}
\phi \\
\chi
\end{array}\right)=\left(\begin{array}{c}
\phi \\
-\chi
\end{array}\right), \\
& I_{0} \Psi_{\mathrm{D}}(r)=\Psi_{\mathrm{D}}(-r)=\left(\begin{array}{c}
\phi(-r) \\
\chi(-r)
\end{array}\right),
\end{aligned}
$$

and thus

$$
I \Psi_{\mathrm{D}}(r)=\left(\begin{array}{c}
\phi(-r) \\
-\chi(-r)
\end{array}\right) .
$$

It implies that if $I \Psi_{\mathrm{D}}(r)=\Psi_{\mathrm{D}}(-r)$, then the large and small components have opposite orbital parities. In fact, it is the case of hydrogenic Dirac wave functions

$$
\Psi=\left(\begin{array}{c}
\phi_{n l j m} \\
\chi_{n \bar{l} j m}
\end{array}\right),
$$

with

$$
\bar{l}=l \pm 1 \quad \text { for } j=l \pm \frac{1}{2} .
$$

Effectively, the main shortcome of the Pauli theory of an electron, as compared to Dirac one, is lack of internal parity, which leads to neglecting the effects of relative parities of the large and small component of $\Psi$ under a projection into $\phi_{\mathrm{P}}$. Still, as it was pointed out by Chatterjee and Lulek [1], the projection operator $R$ in Eq. (16) can be presented (within the approximation up to $k=1$ ), in a general form as

$$
R=R_{0}+\mathrm{i} A \boldsymbol{\sigma} \cdot \boldsymbol{p}+B p^{2}
$$

where $A$ and $B$ are some arbitrary constants. It yields the following two spin-dependent corrections to the Hamiltonian:

$$
\mathcal{H}_{\mathrm{SO}}^{A}=2 A \hbar s \cdot \operatorname{grad} V
$$

and

$$
\mathcal{H}_{\text {SO }}^{B}=\hbar\left(\frac{1}{2 m^{2} c^{2}}+2 A\right) s \cdot(\operatorname{grad} V \times p) .
$$

The latter is the ordinary spin-orbit Hamiltonian of Eq. (14) with a renormalized coupling constant, whereas the former corresponds to the effects of broken internal parity. Putting $A=0$ we achieve only the ordinary term $\mathcal{H}_{\text {So }}^{B}$. In the following we restrict our considerations to this form. 


\section{Form of the spin-orbit coupling in the central and crystalline electric fields}

In the central field of an atom the potential $V$ exhibits the spherical symmetry, i.e. $V(r) \equiv V(r), r=|r|$, so that

$\operatorname{grad} V=\frac{\mathrm{d} V}{\mathrm{~d} r} \frac{\boldsymbol{r}}{r}$

and the spin-orbit interaction operator for a single electron takes on the form

$$
\widehat{\mathcal{H}}_{\text {SO }}=\zeta(r) \widehat{l} \cdot s,
$$

with

$$
\zeta(r)=\frac{\hbar^{2}}{2 m^{2} c^{2}} \frac{1}{r} \frac{\mathrm{d} V}{\mathrm{~d} r}
$$

In the case of an electronic shell $(n l)^{N}$ the Hamiltonian takes the form

$$
\widehat{\mathcal{H}}_{\mathrm{SO}}=\zeta_{n l} \sum_{i=1}^{N} \widehat{l}_{i} \cdot s_{i}
$$

where

$$
\zeta_{n l}=\langle\zeta(r)\rangle_{n l}
$$

is the average over the radial wave functions. For Coulomb potential $V=Z e^{2} / r$, $\zeta_{n l} \sim\left\langle r^{-3}\right\rangle$. Within a single $L S$ Russel-Saunders term, the Hamiltonian (31) reduces to that of Eq. (1).

In the presence of a crystal electric field

$$
V(r)=\sum_{k=0}^{\infty} \sum_{q=-k}^{k} V_{k q}(r) Y_{q}^{(k)}(\theta, \phi),
$$

the form of the spin-orbit interactions has to be modified along the multipole expansion (33). The corresponding expansion in terms of double tensor operators reads $[2,3]$

$$
\mathcal{H}_{\text {SO }}=\sum_{k^{\prime}, k} A^{\left(k^{\prime} 1\right) k} V^{\left(k^{\prime} 1\right) k A_{1}},
$$

where $V^{\left(k^{\prime} 1\right) k A_{1}}$ is the double tensor operator with the orbital rank $k^{\prime}$, spin 1 , the resultant spherical rank $k$, and $A_{1}$ denotes the unit irreducible representation of the point symmetry group of the crystalline field. $A^{\left(k^{\prime} 1\right) k}$ are parameters related to the strength of anisotropic spin-orbit interaction. Their number is equal to the number of appropriate invariants of a given point group, and increases considerably for low symmetries of the crystalline environment of an ion.

In the case of an $f$-electron in a cubic symmetry, the term with $A^{(11) 0}$ corresponds to the spherical spin-orbit interaction, whereas other 5 parameters describe corrections due to the cubic anisotropy (cf. Table I). They are discussed by Kibler and Grenet [8].

Table II presents the corresponding classification for a trigonal crystal field, using the chain of subgroups

$$
D_{3} \subset O \subset S O(3, R) \text {. }
$$


TABLE I

Classification of spin-orbit parameters for $f$-electrons in a crystal field of octahedral symmetry.

\begin{tabular}{c|ll|ll|ll}
\hline \hline$k^{\prime}$ & 1 & & 3 & 5 & \\
\hline$k$ & 0 & 2 & 2 & 4 & 4 & 6
\end{tabular}

TABLE II Classification of spin-orbit parameters for $f$-electrons in a trigonal crystal field, with the intermediate cubic representation.

\begin{tabular}{|c|c|c|c|c|c|c|c|c|c|c|c|}
\hline$k^{\prime}$ & 1 & 3 & & & & 5 & & & & & \\
\hline$\overline{\Delta_{c}^{\prime}}$ & $T_{1}$ & $A_{2}$ & $T_{1}$ & & $T_{2}$ & $T_{1} a$ & & $b$ & & $E$ & $T_{2}$ \\
\hline$\Delta^{\prime}$ & $A_{2} \quad E$ & $A_{2}$ & $A_{2}$ & $E$ & $E$ & $A_{2}$ & $E$ & $A_{2}$ & $E$ & $E$ & $E$ \\
\hline comment & $\lambda_{\|} \quad \lambda_{\perp}$ & new & $\lambda_{\|}^{3}$ & $\lambda_{\perp}^{3}$ & new & $\lambda_{\|}^{5}$ & $\lambda_{\perp}^{5}$ & $\lambda_{\|}^{5}$ & $\lambda_{\perp}^{5}$ & new & new \\
\hline
\end{tabular}

Appropriate double tensor operators, adapted to this chain, are denoted by

$$
V\left(k^{\prime} \Delta_{c}^{\prime} \Delta^{\prime},(1) T_{1} \Delta^{\prime}\right) A_{1},
$$

where $\Delta_{c}^{\prime}$ is an irreducible representation of the cubic group $O$, corresponding to the spherical orbital rank $k^{\prime}$, and $\Delta^{\prime}$ is its counterpart in the trigonal symmetry group $D_{3}$. Some of these parameters can be looked at as simple generalizations of $\lambda$ in Eq. (1) to the $\lambda_{\|}$and $\lambda_{\perp}$, corresponding to the $z$ and $x y$ positions, respectively, but some other are essentially new forms of anisotropic spin-orbit coupling [9-11].

In general, anisotropic spin-orbit parameters are so small in comparison with the crystal field contributions $V_{k q}$, that they have rather little influence on the structure of energy spectrum. Exceptional cases are $s$-state ions, in particular those with the half-filled shell $[3,12]$, and some orbital singlets $[10,12]$ and doublets [13], where the ordinary crystal field does not contribute to the splitting in the first order. Also some triplets can help in distinguishing between $\lambda_{\|}$and $\lambda_{\perp}$ parameters [9].

\section{Final remarks}

We have reviewed some considerations related to the spin-orbit interaction. This interaction arises as a compensating term accompanying the reduction of the Dirac theory of an electron in an external electric field to the theory of Pauli. The main simplification of the Pauli theory is the reduction from four to two components of the wave function, which excludes the internal parity and cancels the effects of relative parities of the large and small components of the Dirac states. We have pointed out after [1] that the very known formula, used here in Eqs. (1), (14), (26), (29), (31) or (34), is not the only possible form of 
spin-dependent corrections to the Pauli Hamiltonian. Clearly, it has proved a posteriori to be an adequate term for a proper account of the fine structure of all known atomic spectra. It is still an open question whether there is another important spin-dependent term, $\mathcal{H}_{\text {SO }}^{B}$ given by Eq. (27). It may be relevant in, say, multicentre electric field in systems without the centre of inversion.

We have also listed anisotropy parameters of the spin-orbit coupling in a crystalline field, and pointed out their role in determinatition of energy spectra of paramagnetic ions in crystals.

\section{Acknowledgments}

The author would like to thank to Prof. Legendziewicz and to Prof. Stręk for giving him an opportunity to have a lecture on the Winter Workshop on Spectroscopy and Structure of Rare Earth Systems.

\section{References}

[1] R. Chatterjee, T. Lulek, Acta Phys. Pol. A 56, 205 (1979).

[2] T. Lulek, Acta Phys. Pol. A 36, 551 (1969); A 40, 797 (1971).

[3] T. Lulek, Acta Phys. Pol. A 43, 705 (1973); A 48, 669 (1975).

[4] J.M. Lévy-Leblond, in: Galilei Group and Galilean Invariance, in series Group Theory and Its Applications, Vol. II, Ed. E.M. Loebl, Academic Press, New York 1971.

[5] V.J. Fushchich, G.A. Nikitin, V.A. Salogub, Rep. Math. Phys. 13, 175 (1978).

[6] L.H. Thomas, Nature 117, 514 (1926); Philos. Mag. VII 3, 1 (1927).

[7] J.D. Jackson, Classical Electrodynamics, sec. ed., Wiley and Sons, New York 1975.

[8] M. Kibler, G. Grenet, Int. J. Quantum Chem. 29, 485 (1986).

[9] T. Lulek, Phys. Status Solidi B 69, 439 (1973).

[10] T. Lulek, Acta Phys. Pol. A 48, 657 (1975).

[11] T. Lulek, G. Kamieniarz, E. Szczepaniak, Acta Phys. Pol. A 49, 111 (1976).

[12] T. Lulek, Phys. Status Solidi B 39, K105 (1970).

[13] B. Lulek, Acta Phys. Pol. A 75, 395 (1989). 